\title{
Quantification of Captopril in Urine Through Surface-Assisted Laser Desorption/Ionization Mass Spectrometry Using 4-Mercaptobenzoic Acid-Capped Gold Nanoparticles as an Internal Standard
}

\author{
Wen-Tsen Chen, Cheng-Kang Chiang, Yang-Wei Lin, and \\ Huan-Tsung Chang \\ Department of Chemistry, National Taiwan University, Taipei, Taiwan
}

We have developed a new internal standard method for the determination of the concentration of captopril (CAP) through surface-assisted laser desorption/ionization mass spectrometry (SALDIMS) using gold nanoparticles (Au NPs). This approach provided linearity for CAP over the concentration range $2.5-25 \mu \mathrm{M}\left(R^{2}=0.987\right)$, with a limit of detection (signal-to-noise ratio $\left.=3\right)$ of $1.0 \mu \mathrm{M}$. The spot-to-spot variations in the concentration of CAP through SALDI-MS analyses performed in the absence and presence of the internal standard were $26 \%$ and $9 \%$, respectively (15 measurements). This approach provides simplicity, accuracy, precision, and great reproducibility to the determination of the levels of CAP in human urine samples. (J Am Soc Mass Spectrom 2010, 21, 864-867) (c) 2010 American Society for Mass Spectrometry

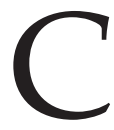
methylpr

(CAP),

1-[(2S)-3-mercapto-2propionyl]-1-proline, is an angiotensinheart failure [1]. Several methods provide good sensitivity and reproducibility for the determination of the concentration of CAP; they, however, require lengthy analyses, high costs, and complicated sampling processes $[2,3]$.

When performing matrix-assisted laser desorption/ ionization mass spectrometry (MALDI-MS) using nanoparticles (NPs), this approach is often called surfaceassisted laser desorption/ionization mass spectrometry (SALDI-MS) [4-7]. Several NPs, including Au [8-11], $\mathrm{Ag}[12], \mathrm{SiO}_{2}[13,14], \mathrm{TiO}_{2}[15,16]$, and $\mathrm{Fe}_{3} \mathrm{O}_{4}[17,18]$ NPs have been applied in SALDI-MS. Although SALDI-MS provides low variation in the shot-to-shot intensities of MS signals for small solutes (30\%; cf. $60 \%$ for MALDI-MS), its practical use for the quantitative determination of small solutes in biological samples remains questionable. Expensive and isotope-based labeling reagents are common internal standards for accurate determination of the concentrations of analytes through MS [19, 20].

We developed a simple and rapid internal standard method-SALDI-MS analysis using 4-mercaptobenzoic

Address reprint requests to Dr. H.-T. Chang, Department of Chemistry, National Taiwan University, 1, Section 4, Roosevelt Road, Taipei 106, Taiwan. E-mail: changht@ntu.edu.tw acid (MBA)-modified Au NPs (MBA-Au NPs) as an internal standard-for the quantitative determination of CAP in urine. We investigated the roles played by the surface density of MBA on each $\mathrm{Au} \mathrm{NP}$, the $\mathrm{pH}$ of the solution, and the salt concentration in determining the sensitivity for the analytical procedure. Our proposed method was validated through the quantitative determination of the concentration of CAP in urine.

\section{Experimental}

\section{Chemicals}

Citric acid, CAP, rhodamine 6G (R6G), salicylazosulfapyridine (SASP), and sodium tetrachloroaurate(III) dehydrate were obtained from Sigma (St. Louis, MO, USA). Mercaptosuccinic acid (MSA), 4-MBA, 11-mercaptoundecanoic acid (11-MUA), 16-mercaptohexadecanoic acid (16-MHA), thioglycolic acid (TA), and trisodium citrate were purchased from Aldrich (Milwaukee, WI, USA). Acetonitrile $(\mathrm{ACN})$ and ammonium hydroxide $\left(\mathrm{NH}_{4} \mathrm{OH}\right)$ were obtained from Acros (Geel, Belgium). Citric acid (100 mM) and $\mathrm{NH}_{4} \mathrm{OH}(25 \%-30 \%)$ were used to prepare ammonium citrate solutions $(0.05-2 \mathrm{mM}, \mathrm{pH}$ $4.00-8.00)$.

\section{Analysis of CAP in Urine}

The 14-nm-diameter Au NPs were prepared [10] and the surface density of thiols on each Au NP was 
determined according to a procedure described previously [21, 22]. Using a calibration curve (fluorescence against the concentration of CAP or MPA), we determined the number of CAP and MPA on each Au NP to be 1750 and 3000, respectively [22]. The urine samples were filtered through $0.45-\mu \mathrm{m}$ membranes and then spiked with CAP to obtain final concentrations of 50 or $200 \mu \mathrm{M}$. Aliquots of the standard solutions of CAP $(0-750 \mu \mathrm{M}, 40.0 \mu \mathrm{L})$ were added to the urine sample $(160 \mu \mathrm{L})$. The spiked urine samples $(200 \mu \mathrm{L})$ were then mixed with $80 \%$ ACN $(800 \mu \mathrm{L})$ and left for $30 \mathrm{~min}$. The proteins in the urine sample were removed through centrifugation at 10,000 rpm for $10 \mathrm{~min}$. The ACN in the suspension solutions $(950 \mu \mathrm{L})$ was evaporated under vacuum and the resulting pellets were re-dissolved in ammonium citrate $(0.1 \mathrm{mM}, \mathrm{pH} 6.00)$ to a final volume of $200 \mu \mathrm{L}$. The Au NPs (final concentration: $7.5 \mathrm{nM}$ ) were added to the protein depleted urine samples (50 $\mu \mathrm{L})$ and the mixtures incubated at room-temperature for $40 \mathrm{~min}$ before SALDI-MS analysis. CAP molecules formed $\mathrm{Au}-\mathrm{S}$ bonds to the Au NPs; hereafter these solutions are referred to as "CAP-Au NP solutions."

The detection of CAP in solutions containing CAP (26.25 $\mu \mathrm{M})$ and 14-nm Au NPs (0.15-75 nM) through SALDI-MS was performed at different concentrations $(0.05-2 \mathrm{mM})$ of ammonium citrate and $\mathrm{pH}$ values $\mathrm{pH}$ $4.00,6.00,8.00)$. When applying the internal standard, MBA $(225 \mu \mathrm{M}, 50 \mu \mathrm{L})$ was added separately to samples of the 14-nm Au NPs (75 nM, $50 \mu \mathrm{L})$ in ammonium citrate solution $(0.125 \mathrm{mM}, \mathrm{pH} 6.00,400 \mu \mathrm{L})$. The mixture was equilibrated for $30 \mathrm{~min}$ to obtain the MBA-Au NP internal standard solutions $(0.5 \mathrm{~mL})$. Equal volumes $(50 \mu \mathrm{L})$ of the MBA-Au NP internal standard and CAP-Au NP solutions were mixed by vortexing for $1 \mathrm{~min}$. Aliquots $(1.0 \mu \mathrm{L})$ of mixtures were pipetted onto a stainless-steel 96-well MALDI target and dried in air at room temperature for $1 \mathrm{~h}$ before SALDI-MS measurements. Mass spectra were recorded in the negative ion mode using a Microflex MALDITOF mass spectrometer (Bruker Daltonics, Bremen, Germany) [10].

\section{Results and Discussion}

To improve the accuracy and precision for the determination of CAP through SALDI-MS using Au NPs (7.5 $\mathrm{nM})$, we tested five compounds as candidates for internal standards: 4-MBA, MSA, 16-MHA, 11-MUA, and TA (each concentration: $22.5 \mu \mathrm{M}$ ). All of these potential internal standards bound readily to the surfaces of the $\mathrm{Au}$ NPs through Au-S bonding, thereby minimizing their desorption during sample preparation. Because 16-MHA and TA were undetectable at $15 \mu \mathrm{M}$, they were unsuitable candidates. Background interference from ionic $\mathrm{Au}$ clusters occurred when using MSA and 11MUA. Under laser irradiation, Au atoms on the Au NP surfaces desorbed and ionized to form ionic Au clusters. MBA provided the lowest background noise and the strongest MS signal; therefore, it became our choice for use as the internal standard for the determination of CAP.

Figure 1 displays SALDI mass spectra of CAP in the presence of MBA when using Au NPs. The spectrum of MBA and CAP clearly displays peaks at $m / z 153.00$ and 216.01, respectively. Because the $\mathrm{pH}$ of the solution affects the charge densities of CAP and Au NPs, it is an important parameter determining their mutual interactions and the desorption/ionization efficiency of CAP (Figure 2a). Upon increasing the $\mathrm{pH}$ value from 4.00 to 6.00 , the intensity of the MS signal of CAP increased, mainly because of the increased negative charge density on the Au NP surfaces $\left(\mathrm{pKa}_{2}\right.$ and $\mathrm{pKa}_{3}$ for citric acid are 4.40 and 5.70, respectively) and CAP (pKa 3.70). At values of $\mathrm{pH}$ greater than 6.00 , the intensity of the MS signal of CAP decreased upon increasing the $\mathrm{pH}$, mainly because of ionization suppression due to the increased ionic strength in the system. The intensity of the MS signal for CAP increased upon increasing the concentration of ammonium citrate from 0.05 to 0.1 $\mathrm{mM}$, due probably to the formation of $\mathrm{NH}_{4}{ }^{+} / \mathrm{CAP}$ ion pairs and to the improved stability of the CAPadsorbed $\mathrm{Au}$ NPs (i.e., the greater surface area of the monodisperse Au NPs resulted in a greater number of CAP molecules being accessible to ionization during laser irradiation). The ionization efficiency of CAP decreased, however, upon increasing the concentration of ammonium citrate to greater than $0.1 \mathrm{mM}$, primarily because of ionization suppression effects and the decreased number of CAP molecules adsorbed on the Au NPs. For the $0.1,0.5$, and $1 \mathrm{mM}$ ammonium citrate solutions ( $\mathrm{pH}$ 6.00), 1750, 1500, and 1000 molecules of $\mathrm{CAP}$, respectively, were bound to each $\mathrm{Au} \mathrm{NP}$ at saturation. The intensity of the CAP signal was higher in $0.1 \mathrm{mM}$ ammonium citrate solution ( $\mathrm{pH}$ 6.00).

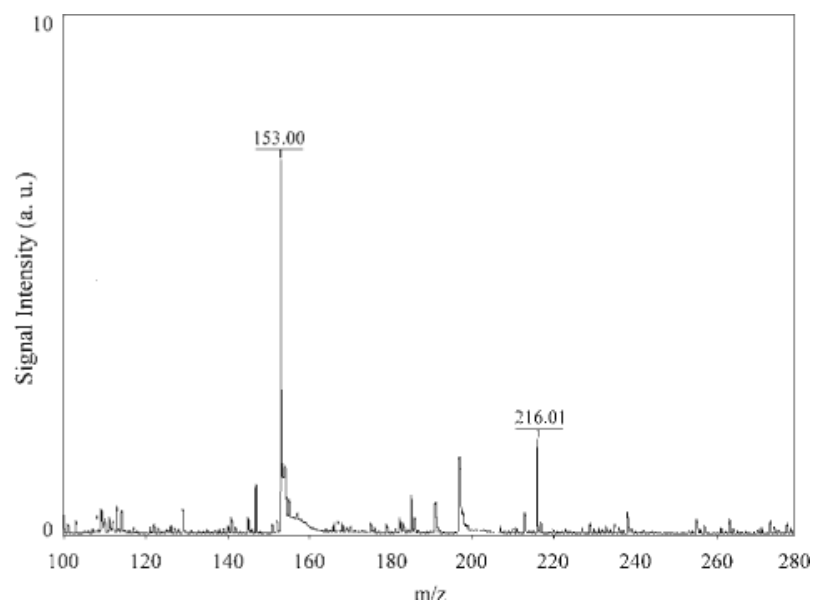

Figure 1. SALDI Mass spectrum of CAP in the presence of MBA. The concentration of each analyte was $22.5 \mu \mathrm{M}$. Solution of 14- $\mathrm{nm}$ Au NPs (7.5 nM) was prepared in $0.1 \mathrm{mM}$ ammonium citrate ( $\mathrm{pH}$ 6.00). The peaks at $m / z 153.00$ and 216.01 correspond to the $[\mathrm{MBA}-\mathrm{H}]-$ and $[\mathrm{CAP}-\mathrm{H}]^{-}$ions, respectively. A total of 150 pulsed laser shots were applied under a laser fluence set at $51.25 \mu \mathrm{J}$. 

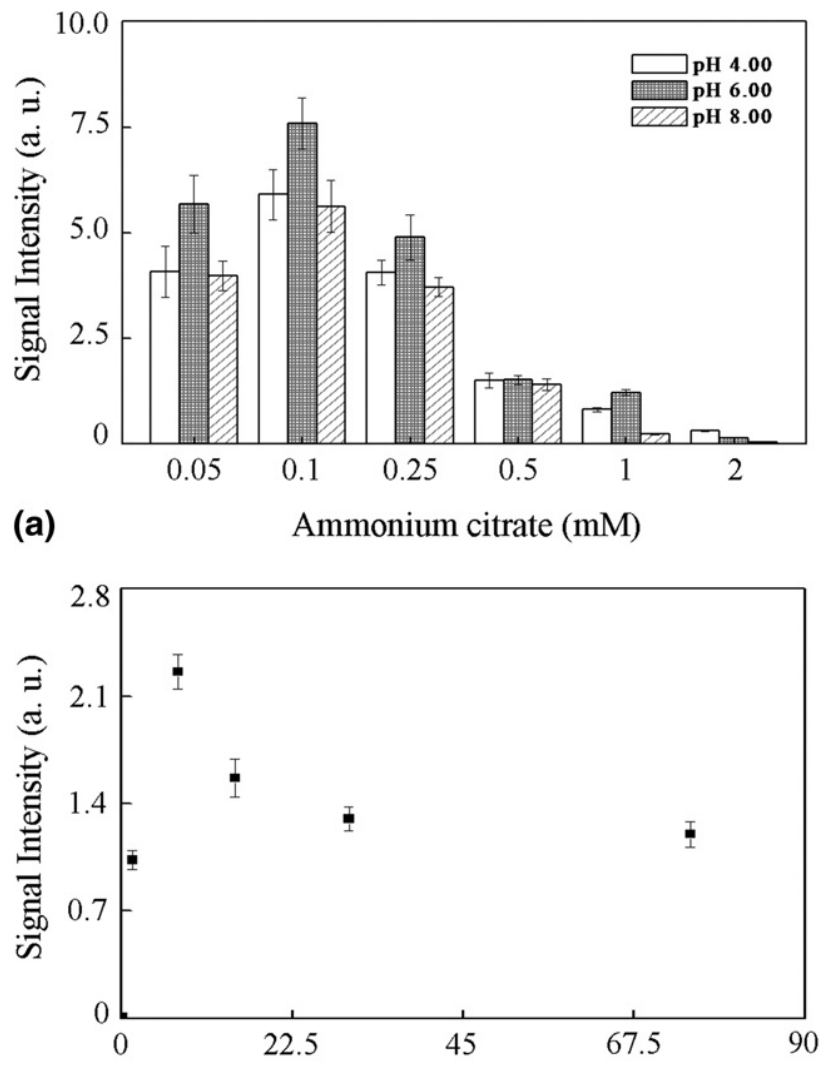

(b)

[AuNPs] (nM)

Figure 2. SALDI-MS signals of CAP in solutions (a) at various concentrations of ammonium citrate and $\mathrm{pH}$ values, and (b) at various concentrations of $\mathrm{Au}$ NPs. In (a), the concentration of $\mathrm{Au}$ NPs was $15 \mathrm{nM}$. In (b), the Au NPs were prepared in $0.1 \mathrm{mM}$ ammonium citrate solution ( $\mathrm{pH}$ 6.00). Other conditions were the same as those described in Figure 1.

We also investigated the role that the concentration of the Au NPs $(0.15-75 \mathrm{nM})$ played in affecting the determination of the concentration of CAP $(26.25 \mu \mathrm{M})$. Figure $2 \mathrm{~b}$ indicates that the intensity of the signal for CAP was maximized when using a $7.5 \mathrm{nM}$ solution of the Au NPs; at lower concentrations, the intensity was lower because of inefficient ionization/desorption of the analytes. Loss of mass-resolution and stronger background signals became problematic when the concentration of Au NPs was greater than $7.5 \mathrm{nM}$ [10]. Under the optimal conditions (7.5 nM Au NPs), we tested the reproducibility for the determination of CAP through SALDI-MS using MBA-Au NPs as the internal standard. To minimize the adsorption of CAP onto the MBA-Au NPs, we prepared the MBA-Au NPs in 22.5 $\mu \mathrm{M}$ MBA solution. A fluorescence result (data not shown) shows that the surfaces of the Au NPs (15 nM) were saturated with 45 and $26.25 \mu \mathrm{M} \mathrm{MBA}$ and CAP, respectively.

Under the optimal conditions (7.5 nM Au NPs; 0.1 $\mathrm{mM}$ ammonium citrate; and $\mathrm{pH}$ 6.00), we determined the concentrations of CAP in solutions though SALDI-
MS. The intensity of the MS signal of CAP and the MS signal ratio of CAP to MBA were both linear against the CAP concentrations over the range from 2.5 to $25 \mu \mathrm{M}$ $\left(R^{2}=0.987\right)$. The limits of detection (LODs) for CAP in the absence and presence of the MBA-Au NP internal standard at an $\mathrm{S} / \mathrm{N}$ ratio of 3 were both $1.0 \mu \mathrm{M}$. In addition, the variability of the signal intensities of CAP from 15 different sample spots at different CAP concentrations $(5,10$, and $20 \mu \mathrm{M})$ decreased when applying the internal standard; the spot-to-spot variations of the signal intensity of CAP were all less than $26 \%$ and $9 \%$ in the absence and presence, respectively, of the internal standard.

To demonstrate the practicality of our internal standard SALDI-MS approach, we determined the concentrations of CAP in urine samples. By using a standard addition method and the internal standard, we detected CAP at concentrations of $47.1 \pm 1.5$ and $192 \pm 3.2 \mu \mathrm{M}$ in urine samples that had been spiked with 50 and 200 $\mu \mathrm{M}$ CAP, respectively $(n=3)$; the recoveries were $94.2 \%$ and $96 \%$, respectively. The loss of CAP was due mainly to the interactions of CAP with the urine matrix, hydrolysis of CAP, and adsorption of CAP on the vials. The RSD values of three replicate inter-day measurements were $7.1 \%, 5.7 \%$, and $8.3 \%$, respectively. Relative to separation techniques with fluorescence detection for the determination of the concentration of CAP in urine samples, our approach is simpler (derivatization of CAP with a fluorophore is not required) and requires shorter analysis time $(<3$ min versus $>30 \mathrm{~min})[2,3]$. This internal standard SALDI-MS approach improves the accuracy and precision of the determination of CAP. It is thus our strong belief that our approach holds great potential for analysis of small molecules in real samples.

\section{Acknowledgments}

The authors acknowledge support for this study by the National Science Council of Taiwan under contracts NSC 98-2113-M-002011-MY3. Y.-W.L. thanks National Taiwan University for PDF support (96R8044).

\section{References}

1. Heel, R. C.; Brogden, R. N.; Speight, T. M.; Avery, G. S. Captopril: A Preliminary Review of Its Pharmacological Properties and Therapeutic Efficacy. Drugs 1980, 20, 409-452.

2. Kusmierek, K.; Bald, E. A Simple Liquid Chromatography Method for the Determination of Captopril in Urine. Chromatographia 2007, 66, 71-74.

3. Tomas, P.-R.; Carmen, M.-L.; Raquel, G. Development and Validation of A Capillary Electrophoresis Method with Laser-Induced Fluorescence Detection for the Determination of Captopril in Human Urine and Pharmaceutical Preparations. Electrophoresis 2006, 27, 2310-2316.

4. Tanaka, K.; Waki, H.; Ido, Y.; Akita, S.; Yoshida, Y.; Yoshida, T.; Matsuo, T. Protein and Polymer Analyses up to $m / z 100,000$ by Laser Ionization Time-of-Flight Mass Spectrometry. Rapid Commun. Mass Spectrom. 1988, 2, 151-153.

5. Sunner, J.; Dratz, E.; Chen, Y.-C. Graphite Surface-Assisted Laser Desorption/Ionization Time-of-Flight Mass Spectrometry of Peptides and Proteins from Liquid Solutions. Anal. Chem. 1995, 67, 4335-4342.

6. Wu, H.-P.; Su, C.-L.; Chang, H.-C.; Tseng, W.-L. Sample-First preparation: A Method for Surface-Assisted Laser Desorption/Ionization Timeof-Flight Mass Spectrometry Analysis of Cyclic Oligosaccharides. Anal. Chem. 2007, 79, 6215-6221. 
7. Najam-ul-Haq, M.; Rainer, M.; Huck, C. W.; Hausberger, P.; Kraushaar, H.; Bonn, G. K. Nanostructured Diamond-Like Carbon on Digital Versatile Disc as a Matrix-Free Target for Laser Desorption/Ionization Mass Spectrometry. Anal. Chem. 2008, 80, 7467-7472.

8. McLean, J. A.; Stumpo, K. A.; Russell, D. H. Size-Selected (2-10 nm) Gold Nanoparticles for Matrix Assisted Laser Desorption Ionization of Peptides. J. Am. Chem. Soc. 2005, 127, 5304-5305.

9. Kawasaki, H.; Sugitani, T.; Watanabe, T.; Yonezawa, T.; Moriwaki, H.; Arakawa, R. Layer-by-Layer Self-Assembled Mutilayer Films of Gold Nanoparticles for Surface-Assisted Laser Desorption/Ionization Mass Spectrometry. Anal. Chem. 2008, 80, 7524-7533.

10. Huang, Y.-F.; Chang, H.-T. Analysis of Adenosine Triphosphate and Glutathione through Gold Nanoparticles Assisted Laser Desorption/ Ionization Mass Spectrometry. Anal. Chem. 2007, 79, 4852-4859.

11. Huang, Y.-F.; Chang, H.-T. Nile Red-Adsorbed Gold Nanoparticle Matrixes for Determining Aminothiols Through Surface-Assisted Laser Desorption/Ionization Mass Spectrometry. Anal. Chem. 2006, 78, 14851493.

12. Chiu, T.-C.; Chang, L.-C.; Chiang, C.-K.; Chang, H.-T. Determining Estrogens Using Surface-Assisted Laser Desorption/Ionization Mass Spectrometry with Silver Nanoparticles as the Matrix. J. Am. Soc. Mass Spectrom. 2008, 19, 1343-1346.

13. Agrawal, K.; Wu, H.-F. Bare Silica Nanoparticles as Concentrating and Affinity Probes for Rapid Analysis of Aminothiols, Lysozyme, and Peptide Mixtures Using Atmospheric-Pressure Matrix-Assisted Laser Desorption/Ionization Ion Trap and Matrix-Assisted Laser Desorption/ Ionization Time-of-Flight Mass Spectrometry. Rapid Commun. Mass Spectrom. 2008, 22, 283-290.

14. Wen, X.; Dagan, S.; Wysocki, V. H. Small-Molecule Analysis with Silicon-Nanoparticle-Assisted Laser Desorption/Ionization Mass Spectrometry. Anal. Chem. 2007, 79, 434-444.
15. Torta, F.; Fusi, M.; Casari, C. S.; Bottani, C. E.; Bachi, A. Titanium Dioxide Coated MALDI Plate for On Target Analysis of Phosphopeptides. J. Proteome Res. 2009, 8, 1932-1942.

16. Lee, K.-H.; Chiang, C.-K.; Lin, Z.-H.; Chang, H.-T. Determining Enediol Compounds in Tea Using Surface-Assisted Laser Desorption/Ionization Mass Spectrometry with Titanium Dioxide Nanoparticle Matrixes. Rapid Commun. Mass Spectrom. 2007, 21, 2023-2030.

17. Chen, W.-Y.; Chen, Y.-C. Affinity-Based Mass Spectrometry Using Magnetic Iron Oxide Particles as the Matrix and Concentrating Probes for SALDI MS Analysis of Peptides and Proteins. Anal. Bioanal. Chem. 2006, 386, 699-704

18. Chen, C.-T. Chen, Y.-C. $\mathrm{Fe}_{3} \mathrm{O}_{4} / \mathrm{TiO}_{2}$ Core/Shell Nanoparticles as Affinity Probes for the Analysis of Phosphopeptides Using $\mathrm{TiO}_{2}$ Surface-Assisted Laser Desorption/Ionization Mass Spectrometry. Anal. Chem. 2005, 77, 5912-5919.

19. Muddiman, D. C.; Gusev, A. I.; Langner, K. S.; Proctor, A.; Hercues, D. M.; Tata, P. Simultaneous Quantification of Cyclosporin A and Its Major Metabolites by Time-of-Flight Secondary-Ion Mass Spectrometry and Matrix-Assisted Laser Desorption/Ionization Mass Spectrometry Utilizing Data Analysis Techniques: Comparison with High-Performance Liquid Chromatography. J. Mass Spectrom. 1995, 30, 1469-1479.

20. Kang, M.-J.; Tholey, A.; Heinzle, E. Quantitation of Low Molecular Mass Substrates and Products of Enzyme Catalyzed Reactions Using MatrixAssisted Laser Desorption/Ionization Time of-Flight Mass Spectrometry. Rapid Commun. Mass Spectrom. 2000, 14, 1972-1978.

21. Huang, C.-C.; Chen, C.-T.; Shiang, Y.-C.; Lin, Z.-H.; Chang, H.-T. Synthesis of Fluorescent Carbohydrate-Protected $\mathrm{Au}$ Nanodots for Detection of Concanavalin A and Escherichia coli. Anal. Chem. 2009, 81, 875-882.

22. Chen, S.-J.; Chang, H.-T. Nile Red-Adsorbed Gold Nanoparticles for Selective Determination of Thiols Based on Energy Transfer and Aggregation. Anal. Chem. 2004, 76, 3727-3733. 